\title{
Determinants and outcomes of access- related blood-stream infections among Irish haemodialysis patients; a cohort study
}

Husham Mohamed ${ }^{1,2}$, Alaa Ali ${ }^{1,2}$, Leonard D. Browne ${ }^{2}$, Nuala H. O'Connell ${ }^{2,3}$, Liam Casserly ${ }^{1,2}$, Austin G. Stack ${ }^{1,2,4^{*}}$ and Wael F. Hussein ${ }^{1,2}$

\begin{abstract}
Background: Infections are the second leading cause of death and hospitalisation among haemodialysis (HD) patients. Rates of access-related bloodstream infections (AR-BSI) are influenced by patient characteristics and local protocols. We explored factors associated with AR-BSI in a contemporary cohort of HD patients at a tertiary nephrology centre.
\end{abstract}

Methods: A retrospective cohort of 235 chronic HD patients was identified from a regional dialysis programme between Jan 2015 and Dec 2016. Data on demographics, primary renal disease, comorbid conditions and dialysis access type were obtained from the Kidney Disease Clinical Patient Management System (KDCPMS). Data on blood cultures were captured from the microbiology laboratory. Poisson regression with robust variance estimates was used to compare infection rates and relative risk of AR-BSI according to the site and type of vascular access.

Results: The mean age was 65 ( \pm 15 ) years, 77\% were men, and the median follow up was 19 months (IQR: 10-24 months), accumulating 2030 catheter-months and 1831 fistula-months. Overall rates of AR-BSI were significantly higher for central venous catheter (CVC) compared to arteriovenous fistula (AVF), (2.22, 95\% (Cl): 1.62-2.97) versus 0.11 (0.01-0.39) per 100 patient-months respectively), with a rate ratio of 20.29 (4.92-83.66), $p<0.0001$. This pattern persisted across age, gender and diabetes subgroups. Within the CVC subgroup, presence of a femoral CVC access was associated with significantly higher rates of AR-BSI (adjusted RR 4.93, 95\% Cl: 2.69-9.01). Older age ( $75+$ versus $<75$ years) was not associated with significant differences in rates of AR-BSI in the unadjusted or the adjusted analysis. Coagulase negative Staphylococcus (61\%) and Staphylococcus aureus (23\%) were the predominant culprits. AR-BSIs resulted in access loss and hospitalisation in 57 and $72 \%$ of events respectively, and two patients died with concurrent AR-BSI.

Conclusions: Rates of AR-BSI are substantially higher in CVC than AVF in contemporary HD despite advances in catheter design and anti-infective protocols. This pattern was consistent in all subgroups. The policy of AVF preference over CVC should continue to minimise patient morbidity while at the same time improving antiinfective strategies through better care protocols and infection surveillance.

Keywords: Haemodialysis, Arteriovenous fistula, Bacteraemia, Catheter infections, Catheter-related bloodstream infections, Access-related bloodstream infections, Central venous catheter

\footnotetext{
* Correspondence: austin.stack@ul.ie

${ }^{1}$ Division of Nephrology, Department of Medicine, University Hospital Limerick, Limerick, Ireland

${ }^{2}$ Graduate Entry Medical School, University of Limerick, Limerick, Ireland

Full list of author information is available at the end of the article
}

(c) The Author(s). 2019 Open Access This article is distributed under the terms of the Creative Commons Attribution 4.0 International License (http://creativecommons.org/licenses/by/4.0/), which permits unrestricted use, distribution, and reproduction in any medium, provided you give appropriate credit to the original author(s) and the source, provide a link to the Creative Commons license, and indicate if changes were made. The Creative Commons Public Domain Dedication waiver (http://creativecommons.org/publicdomain/zero/1.0/) applies to the data made available in this article, unless otherwise stated. 


\section{Background}

Patients on haemodialysis (HD) endure infection rates that are more than 26 times higher than that of the general population [1], and more than 100 to 200 -fold higher for specific organisms [2]. They are the second leading cause of hospitalisation and mortality in the dialysis population [3-5]. National and international guidelines along with national policy initiatives [6-9] recommend the use of arteriovenous fistula (AVF) whenever possible, as the risk of infections and other complications is highest among patients using central venous catheters (CVCs) [3, 10,11]. Despite the dangers associated with $\mathrm{CVC}$ use, these devices remain the principal type of access in the majority of HD patients in Ireland $[12,13]$ and internationally $[14]$.

The alarmingly high rates of access-related bloodstream infections (AR-BSI) in patients undergoing dialysis with a CVC has forced changes in clinical practices that include better anti-infective protocols, increasing adoption of catheter lock solutions, and better antimicrobial surveillance protocols in order to reduce CVC-related infection rates [15-18]. It is unclear, however, to what extent these changes have curbed the high rates of AR-BSI in the context of an increasing elderly HD phenotype with a high burden of complex health problems. It is also uncertain whether any benefit derived from these measures extends to very high-risk groups especially the elderly, patients with diabetes and those dialysed with a femoral CVC. While the formation of a functioning AVF is the preferred vascular access, this is not easily attainable in all individuals, especially elderly patients on HD [19]. Furthermore it remains controversial whether CVCs are superior to AVFs among elderly patients undergoing dialysis with a recent study finding lower rates of catheter-related bacteraemia in elderly patients compared to younger patients [18, 20-22].

Within the Irish health system, data is lacking on the on the frequency and impact of AR-BSI in HD. The availability of such data along with clinical outcomes will help inform healthcare providers and policy-decision makers on access type, and will drive quality improvement initiatives to improve patient outcomes. We determined the rates of AR-BSI in a contemporary cohort of HD patients dialysed with a CVC or AVF and explored the relative contribution of demographic and clinical factors to overall rates of AR-BSI.

\section{Methods}

\section{Study design and setting}

We conducted a retrospective observational study to explore AR-BSI in a contemporary cohort of HD patients. We identified all adult patients receiving chronic HD during 2015 and 2016 under the care of a tertiary nephrology centre. Patients were observed from the first to the last dialysis they received during the period between $1 / 1 / 2015$ and $31 / 12 / 2016$. Primary access type and changes from CVC to AVF or vice versa during the observation period were recorded. All AR-BSI events were captured during the observation period and outcomes of these events were recorded. The rates of BSIs were calculated using standard definitions described below. As this study aimed to examine rates of bacteraemia associated with access types used over prolonged periods of time in outpatient settings, temporary dialysis catheters were not included in the analysis.

\section{Description of local practice}

Patients received dialysis at a unit attached to the main hospital or at an affiliated outpatient dialysis unit. All tunnelled CVCs were inserted by interventional radiologists. CVC type used is ProGuide ${ }^{\mathrm{rn}}$, produced by Merit Medical Systems ${ }^{\circ}$. An access care bundle was in place to reduce risk of infection. This included protocols for hand hygiene and use of protective equipment during connection and disconnection of dialysis lines. BioPatch ${ }^{\circ}$ (Ethicon $\odot$ ) dressings were applied to the exit site, and were changed on a weekly basis. Catheters were locked with $46.7 \%$ citrate. Disposable catheter hubs were used. Before connection, catheter hubs and fistula needle insertion sites were decontaminated with $10 \%$ iodinated povidone. When an access infection was suspected, two sets of blood cultures were taken from each port of a catheter or from a peripheral vessel in case of a fistula. Empiric antimicrobial therapy was commenced when there was strong clinical suspicion after collection of culture samples. Dialysis catheters were removed if the causative organism was Staphylococcus aureus, fungal, or if the infection is difficult to clear. The HD unit protocol mandates regular screening for methicillinresistant $S$. aureus (MRSA), vancomycin-resistant Enterococcus species (VRE), extended spectrum beta lactamase (ESBL)- producing organisms and carbapenemresistant Enterobacteriaceae (CRE). Colonization with MRSA is treated with mupirocin nasal disinfection and chlorhexidine wash for skin disinfection followed by rescreening. All patients colonized with MRSA or CRE receive dialysis in isolation rooms. All inpatient and outpatient microbiology samples from the healthcare region are sent to a single central microbiology laboratory located within the main hospital.

\section{Participants and data sources}

Patients were identified using data from the Kidney Disease Clinical Patient Management System (KDCPMS), a national multi-domain electronic health record system that tracks clinical care of HD patients in the Irish health system. Patients who received acute dialysis or holiday dialysis 
treatments were excluded. Study entry age, access type and access site were defined for each patient at the date of first dialysis during the study period. Baseline data were captured on age, sex, primary cause of End Stage Kidney Disease (ESKD), comorbid conditions, the type and site of the dialysis access. Blood culture results from patients during the observation period were retrieved from the microbiology laboratory database. All changes in dialysis access type and site were recorded during the study period. Access site was recorded as upper extremity or femoral location. The internal jugular vein (IJ) was the most common access at the centre, with subclavian access only reserved for situations where IJ access was not attainable. Due to the small number of non-IJ sites, comparisons between different non-femoral CVC's was not reliable or informative. The determination of infection rates and rate ratios was based on the current access in use at time of infection.

\section{Definition of AR-BSI and calculation of rates}

Access-related bloodstream infection (AR-BSI) was defined as growth of a typical organism with either a documented exit site or tunnel infection, or with no other identified source of infection. Patients with atypical organisms who received antimicrobial treatment for 2 weeks or more were also considered to have AR-BSI. Blood cultures that were positive with the same organism within 21 days of a previous positive culture were considered part of the initial event and not counted as a separate event. The definition of AR-BSI in our study did not include sampling from a peripheral vein (as recommended by CDC), however, previous reports suggest that peripheral blood culture results add little to the sensitivity and specificity of cultures blood obtained from the HD circuit and the venous catheter hub [23].

We followed guidelines issued by the Centers for Disease Control and Prevention (CDC) - National Healthcare Safety Network (NHSN) for calculation of event rates $[24,25]$. The number of chronic HD patients under the care of the tertiary centre on the first day of each month was used as the denominator for that month. Vascular access type at the start of the month was used to identify subgroups for catheter patient-months and fistula patient-months. The numerator for AR-BSI event rates for each month was the number of identified AR-BSI events during that month. All recorded CVCs were tunnelled catheters (none were temporary dialysis catheters). Only two arteriovenous grafts were in use during the observation period. For the purposes of this analysis, these were grouped with AVF.

\section{Ethical approval}

Ethical approval was not sought this study as the surveillance of infections in dialysis patients is part of regular clinical audit and the hospital's quality improvement programme [26].

\section{Statistical analysis}

Baseline characteristics were presented for the whole group and for subgroups of study entry access type. Continuous variables were presented as mean \pm standard deviations and categorical variables were presented as percentages. Comparisons between groups according to vascular access type were performed by analysis of variance for continuous variables and Chi-square test for categorical variables.

Poisson regression employing the Huber-White sandwich variance estimator was used to compare the infection rates and determine the risk of infection according to vascular access type. Rates of AR-BSI were presented as events per 100 patient-months with robust $95 \%$ confidence intervals (CIs). To determine factors associated with bacteraemia among patients using $\mathrm{CVC}$, univariable and multivariable models were constructed to examine the association of demographic and clinical factors, and access insertion site with the risk of AR-BSI. Model development progressed using a manual strategy taking into consideration known associations from published literature, and statistical significant univariable associations. A final model was constructed to determine the association of age, sex, diabetes, and access type with the outcome of AR-BSI in patients receiving dialysis by CVC. Goodness of fit was assessed using the Pearson and Deviance statistics. All analyses were conducted using $\mathrm{R}$ version 3.4.

\section{Results}

Baseline characteristics of study population

A total of 281 adult patients received dialysis between $1 /$ $1 / 2015$ and 31/12/2016. Of those, 46 were excluded (Acute HD: 26, Visitor/Holiday HD: 20) leaving 235 patients eligible for inclusion in the final study sample. During the observation period, the monthly census increased from 134 patients to 181 from Jan 2015 to Dec 2016, and the percentage of patients receiving dialysis by CVC ranged from 48 to $57 \%$ (Additional file 1: Table S1). Median patient follow up was 19 months (IQR: 10-24 months) resulting in 3861 patient months of observation, 2030 catheter-months and 1831 fistulamonths (Additional file 1: Table S2). There were 74 bloodstream infections detected during the observation period, of which 47 were related to HD access (Fig. 1).

Table 1 illustrates the baseline characteristics of participants at study entry. The average age was $65( \pm 15)$ years, $28.5 \%$ were 75 years of age or older, and the 


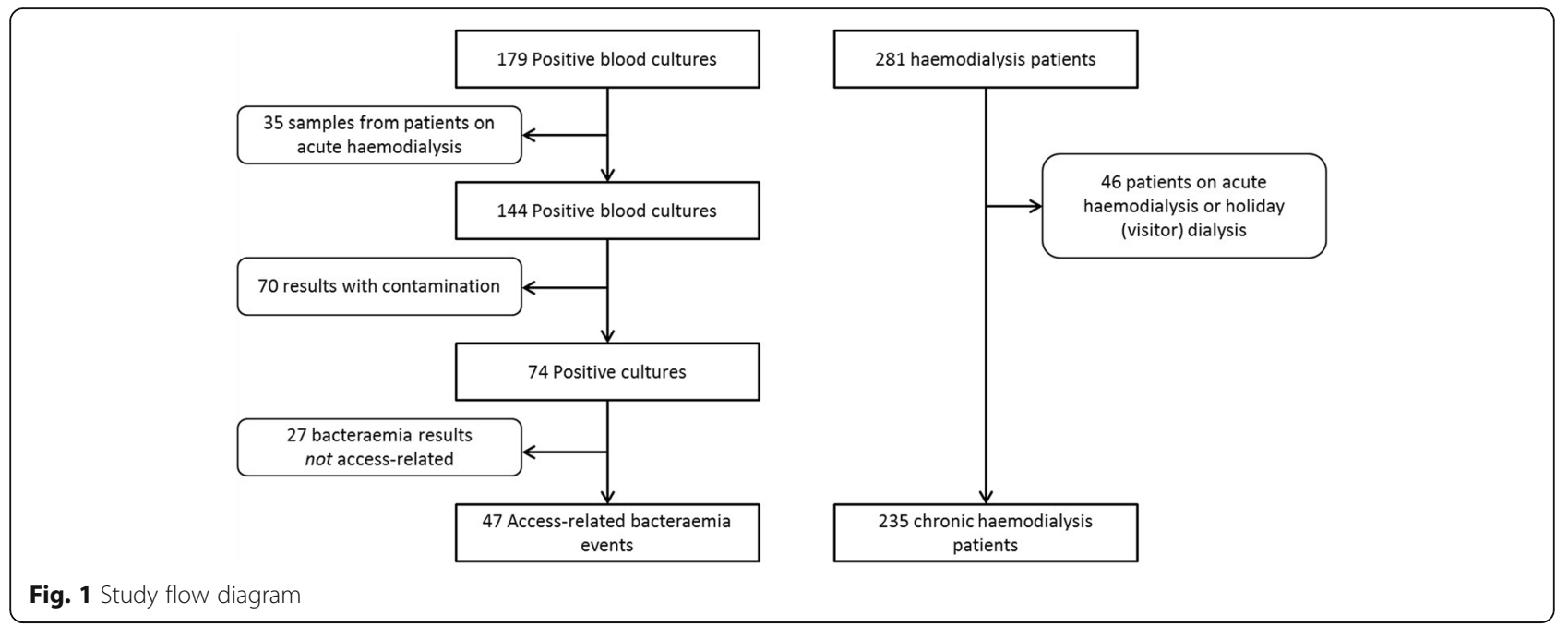

Table 1 Baseline characteristics of the total population

\begin{tabular}{|c|c|c|c|}
\hline & $\begin{array}{l}\text { Overall } \\
n=235 *\end{array}$ & $\begin{array}{l}\text { AVF/AVG at study } \\
\text { entry } * n=96\end{array}$ & $\begin{array}{l}\text { CVC at study } \\
\text { entry } * n=139\end{array}$ \\
\hline Femoral access & 4.3 & 2.1 & 5.8 \\
\hline Age in years (mean (SD)) & $65(15)$ & $66(14)$ & $64(15)$ \\
\hline \multicolumn{4}{|l|}{ Age groups } \\
\hline$<75$ years & 71.5 & 69.8 & 72.7 \\
\hline $75+$ & 28.5 & 30.2 & 27.3 \\
\hline Women* & 33.2 & 22.9 & 40.3 \\
\hline \multicolumn{4}{|c|}{ Primary cause of renal disease* } \\
\hline Diabetes mellitus & 23.8 & 27.1 & 21.6 \\
\hline Glomerulonephritis & 19.6 & 19.8 & 19.4 \\
\hline Cystic kidney disease & 8.1 & 13.5 & 4.3 \\
\hline Other urologic & 6.4 & 6.2 & 6.5 \\
\hline Hypertension & 3.8 & 6.2 & 2.2 \\
\hline Other cause & 16.2 & 11.5 & 19.4 \\
\hline Unknown/missing & 22.1 & 15.6 & 26.6 \\
\hline \multicolumn{4}{|l|}{ Comorbidities } \\
\hline Hypertension & 68.9 & 67.7 & 69.8 \\
\hline Diabetes & 35.3 & 33.3 & 36.7 \\
\hline $\begin{array}{l}\text { Atherosclerotic } \\
\text { heart disease }\end{array}$ & 24.3 & 26.0 & 23.0 \\
\hline $\begin{array}{l}\text { Congestive } \\
\text { heart failure }\end{array}$ & 15.7 & 15.6 & 15.8 \\
\hline Other cardiac & 22.1 & 25.0 & 20.1 \\
\hline $\begin{array}{l}\text { Cerebrovascular } \\
\text { disease }\end{array}$ & 11.9 & 13.5 & 10.8 \\
\hline $\begin{array}{l}\text { Peripheral vascular } \\
\text { disease }\end{array}$ & 8.5 & 7.3 & 9.4 \\
\hline
\end{tabular}

*Column \% unless specified otherwise ${ }^{*} p<0.05$ majority were men (77\%). Diabetes was the most common cause of ESKD, while hypertension and diabetes were the most prevalent recorded comorbid conditions. The distribution of baseline characteristics was similar for patients dialysed by either AVF or CVC with the exception of sex and primary cause of ESKD. The CVC group had significantly higher proportion of women and fewer patients who had diabetes, hypertension and renal cystic disease as their primary cause of renal disease.

The baseline characteristics of patients dialysed through a CVC is shown in Table 2. Patients age $\geq 75$ years had significantly more hypertension and congestive heart failure than younger counterparts while the distribution of other characteristics was similar. Distribution of baseline characteristics in the whole group (any access) by age group is shown in Additional file 1: Table S3.

\section{Rates of access-related bloodstream infection (AR-BSI) in CVC versus AVF}

The rate of AR-BSI in patients dialysed with a CVC was significantly higher than in patients with an AVF [2.22 (95\% 1.62-2.97) versus 0.11 (95\% CI: 0.01-0.39) per 100 patient months respectively, $p<0.001$, with an unadjusted incidence rate ratio of 20.29 (95\% CI $4.92-$ 83.66) as shown in Fig. 2. Among all specified subgroups of age, sex, diabetes and access site, the incidence rates of AR-BSIs were significantly and substantially higher among those using a CVC compared to AVF.

By far the highest rate of AR-BSI was observed with a femoral CVC access, 8.50 (95\% CI 4.52-14.53) events per 100 patient months. Among patients receiving dialysis by tunnelled catheter, the rate ratio of AR-BSI for femoral versus non-femoral CVC was 4.98 (95\% CI 2.71-9.15), $p<0.001$. In multivariable analysis adjusting for age, sex, and diabetes, use of a femoral CVC was associated with 4.93 times (95\% CI 2.69-9.01) the risk of infection compared to a non-femoral CVC site - Fig. 3. 
Table 2 Baseline characteristics by age group among patients with a tunnelled central venous catheter at study entry

\begin{tabular}{|c|c|c|c|}
\hline \multirow[t]{2}{*}{ Characteristic } & \multirow{2}{*}{$\begin{array}{l}\text { All patients } \\
(n=139)\end{array}$} & \multicolumn{2}{|c|}{ Age groups (years) } \\
\hline & & $<75(n=101)$ & $75+(n=38)$ \\
\hline Femoral access & 5.8 & 5.9 & 5.3 \\
\hline Age in years $(\text { mean }(S D))^{*}$ & $64(15)$ & $57(12)$ & $82(5)$ \\
\hline Female & 40.3 & 38.6 & 44.7 \\
\hline \multicolumn{4}{|l|}{ Primary cause of renal disease } \\
\hline Diabetes mellitus & 21.6 & 20.8 & 18.4 \\
\hline Glomerulonephritis & 19.4 & 19.8 & 18.4 \\
\hline Cystic kidney disease & 4.3 & 5.0 & 2.6 \\
\hline Other urologic & 6.5 & 6.9 & 5.3 \\
\hline Hypertension & 2.2 & 3.0 & 0.0 \\
\hline Other cause & 19.4 & 19.8 & 18.4 \\
\hline Unknown/missing & 26.6 & 24.8 & 31.6 \\
\hline \multicolumn{4}{|l|}{ Comorbidities } \\
\hline Hypertension* & 69.8 & 62.4 & 89.5 \\
\hline Diabetes & 36.7 & 34.7 & 42.1 \\
\hline $\begin{array}{l}\text { Atherosclerotic } \\
\text { heart disease }\end{array}$ & 23.0 & 18.8 & 34.2 \\
\hline Congestive heart failure* & 15.8 & 10.9 & 28.9 \\
\hline Other cardiac & 20.1 & 15.8 & 31.6 \\
\hline Cerebrovascular disease & 10.8 & 8.9 & 15.8 \\
\hline $\begin{array}{l}\text { Peripheral vascular } \\
\text { disease }\end{array}$ & 9.4 & 9.9 & 7.9 \\
\hline
\end{tabular}

There were no significant differences in the rates of AR-BSI by age ( $75+$ versus $<75$ years) in the unadjusted or the adjusted analyses.

\section{Type of organism}

The distribution of organisms isolated from blood cultures is shown in Table 3. The most common isolates were Staphylococci identified in $85 \%$ of positive blood cultures. The majority of these were Coagulase-negative Staphylococcal (CoNS) infections with S. aureus contributing $23.4 \%$. No fungal infections were recorded during the study period.

\section{AR-BSI outcomes}

AR-BSIs resulted in hospitalisation and access loss in 34 (72\%) and 27 (57\%) of the events respectively. Two patients died with AR-BSI events; one with poly-microbial infection.

\section{Sensitivity analysis}

To address whether a number of individuals prone to risk in the CVC group may be leading to an exaggeration of risk, we conducted a sensitivity analysis by excluding patients with more than 1 and more than 2 recorded CRBSIs (Catheter-Related Bloodstream Infections). No variables were found to be statistically significant when excluding patients with more than 1 case of CBRSI $(n=10)$. However, excluding patients with more than two cases $(n=2)$ yielded similar results to that of the primary analysis. The median duration between CRBSI's among these patients was 117 days with a minimum of 50 days and a maximum of 465 days between events. There were a few patients with recurrent infections (more than 2 cases of CBRSIs) in this study and omission of these patients did not alter the primary findings of this study.

\section{Discussion}

In this large centre-based study, we emphasise the significant risk of bloodstream infections associated with use of tunnelled dialysis catheters. Compared to patients who were using an AVF, patients with a CVC experienced a 20-fold higher risk of access-related bacteraemia. The risk associated with CVC use was independent of age and comorbid disease measured at baseline. Subgroup analysis confirmed that the pattern of risk from CVC was present in younger and in older patients, men and women, and in patients with and without diabetes. These results would suggest that despite advances in anti-infective protocols, innovative catheter designs, and the implementation of national guidelines, CVCs remain a major source of serious morbidity in HD patients.

The adverse impact of CVC over AVF on catheterrelated bacteraemia rates was overwhelmingly apparent from this analysis. Our observed rates of AR-BSI events were 2.22 and 0.11 events per 100 patient-months for CVC and AVF respectively, a 20-fold difference. Our findings are concordant with reports from other parts of the world. AR-BSI rates in patients with CVC and AVF were 3.1 and 0.6 , and 3.5 and 1.7 per 100 patientmonths from Greece and Brazil respectively [4, 18]. A study from the National Healthcare Safety Network (NHSN) in the US reported pooled rates of 2.55 and 0.23 events per 100 patient-months for CVC and AVF respectively from 2007 and 2011 [27], while a more recent report suggested improvements with estimates of 1.83 and 0.16 for CVCs and AVF respectively [24]. The patient-months distribution in this last report was $19 \%$ for CVC and $63 \%$ for AVF, reflecting a much lower dependence on tunnelled catheters than in our Irish cohort. The rates of AR-BSI in our cohort compare favourably with those from the CDC report in the US [24] in that rates were at the 50th percentile for CVC-related BSIs, and below the 25th for AVF-related infections. Despite these reassuring statistics, there is emerging evidence that further improvements are 


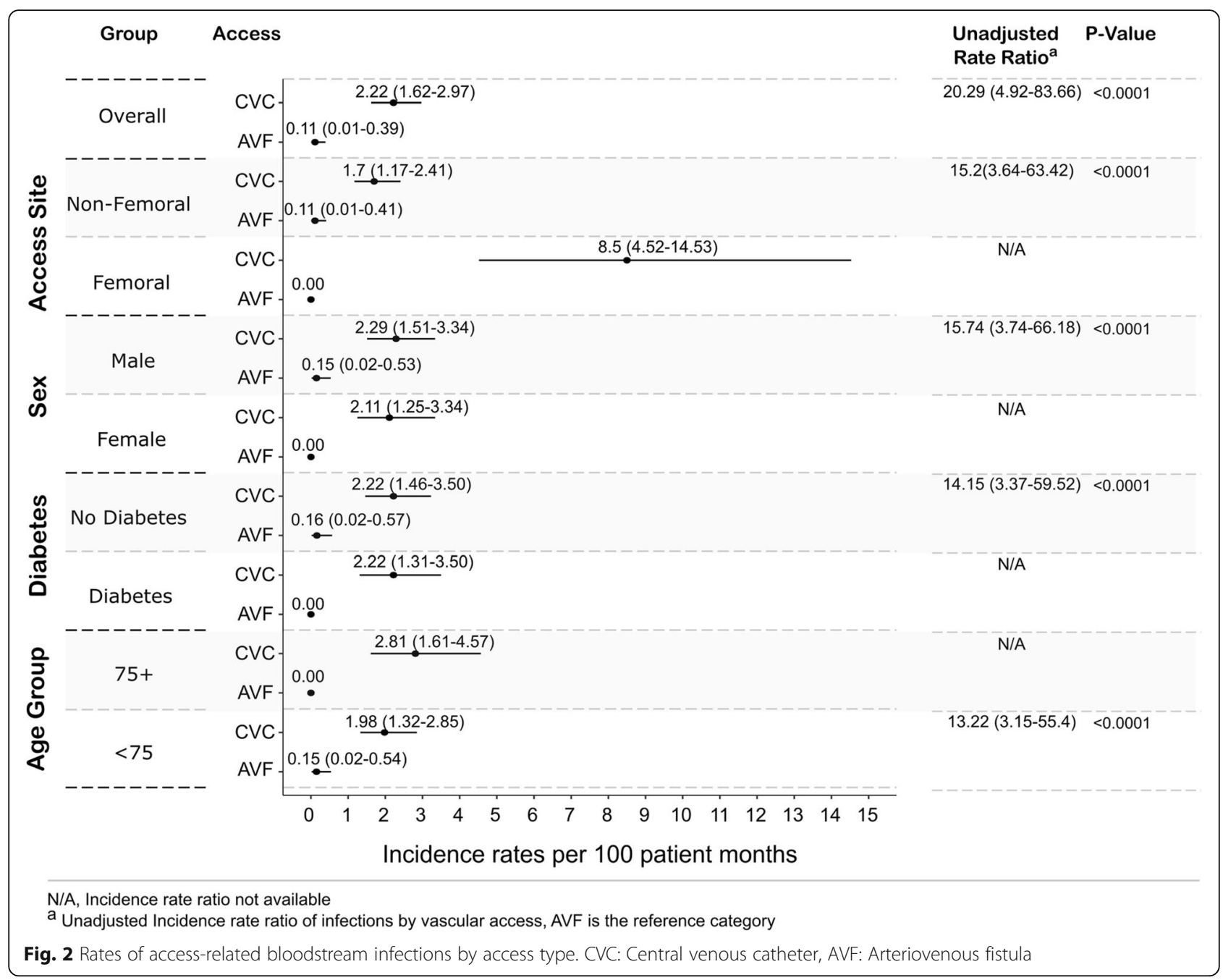

possible. Hymes et al, reported significant reductions in AR-BSI to 0.67 per 100 patient-months with the introduction of antimicrobial barrier caps [28]. A further study by the CDC Dialysis BSI Prevention Collaborative, demonstrated a sustained reduction in CVC-related BSI's from 2.26 to 1.08 events per 100 patient-months using a bundle of BSI-preventative interventions [29]. These encouraging findings suggest that there is further scope to reduce infection rates associated with CVC use and emphasise the need for sustained quality improvement initiatives.

Controversy currently exists as to whether tunnelled dialysis catheters should be considered a satisfactory access type for dialysis in older patients [30]. A lower rate of complications in older patients would support this approach. In support of this hypothesis, Murea et al found lower rates of catheter-related bacteraemia in patients above 75 years versus younger patients (1.67 versus 5.99 events per 100 patient-months respectively, HR 0.33 (95\% CI 0.20-0.55) [17], citing lower rates of nasal colonisation, less sweating, and less mechanical stress on the catheter as potential reasons. Wang et al showed similar results [16]. However, several studies have found no association between age and AR-BSIs [18, 20-22]. Furthermore, mortality risks (infectionrelated, cardiovascular-related, and all-cause) are higher in patients on dialysis by CVC even in elderly patients [31,32]. The findings from our study are in direct contrast with those of Murea et al in that elderly patients experienced risks that were similar to those of younger patients.

The most significant factor associated with increased catheter-related BSIs in our population was site of the tunnelled dialysis catheter. In univariate and multivariable analysis, femoral access was associated with a fivefold increase in the rate of AR-BSI when compared to non-femoral access. This observation may relate to greater levels of skin contamination at the femoral area, relatively more difficult access for cleaning and observation, or may relate to some patient characteristics such 


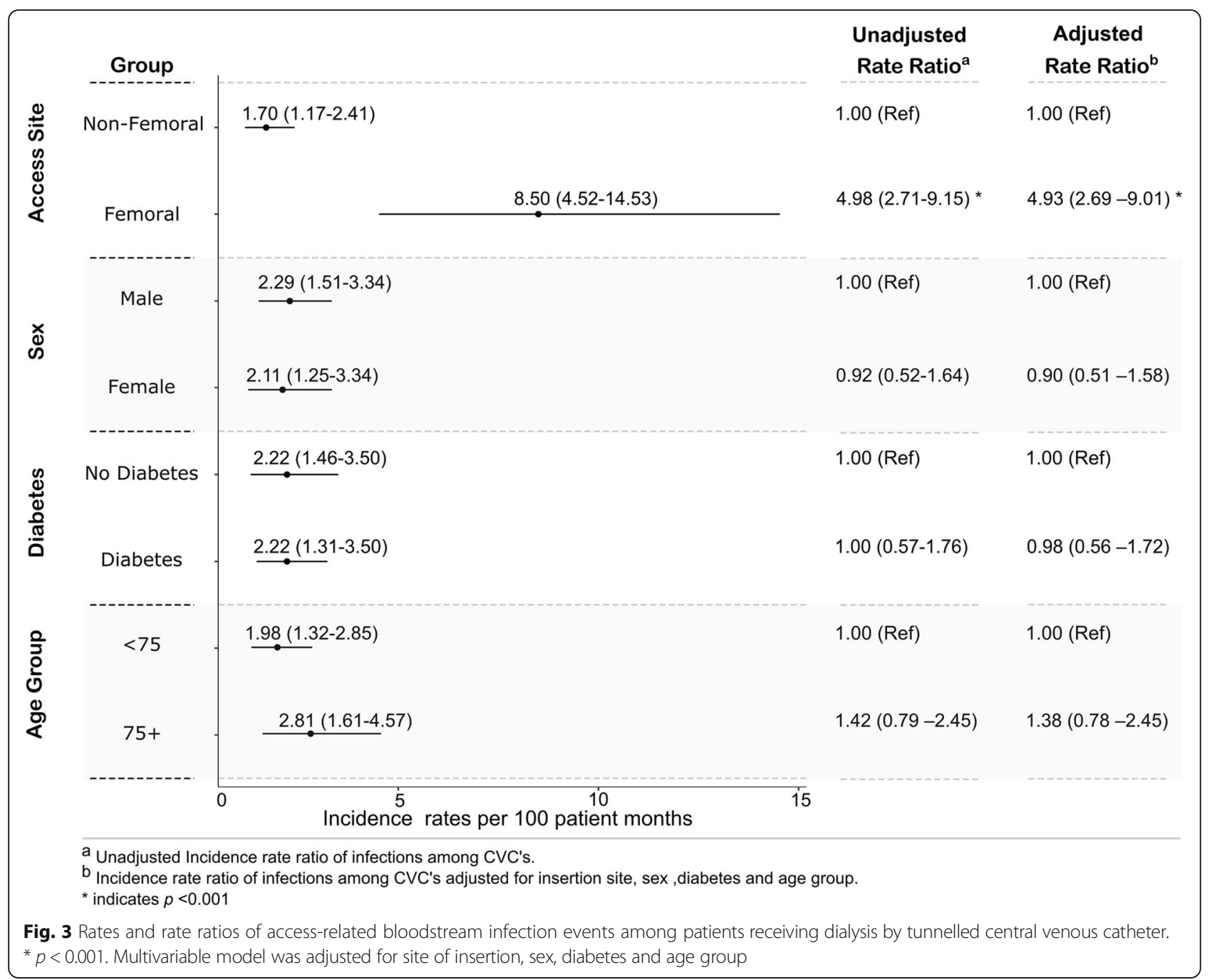

Table 3 Types of organisms isolated in access-related bloodstream infections

\begin{tabular}{ll}
\hline Organism & Frequency (\%) \\
\hline Coagulase-negative Staphylococcus & 61.7 \\
Staphylococcus aureus & 23.4 \\
$\quad$ MSSA: 19.1\% & \\
MRSA: 4.3\% & \\
Beta-haemolytic Streptococci & 4.3 \\
Streptococcus parasanguinis & 2.1 \\
Enterococcus spp. & 2.1 \\
Vancomycin-resistant enterococci (VRE) & 2.1 \\
Proteus spp. & 2.1 \\
Poly-microbial & 2.1 \\
\hline
\end{tabular}

as vintage or poor health. Femoral access is known to have higher rates of complications overall, including infection and malfunction [16, 17]. Quality improvement programs need to focus on this high-risk group of patients. We did not observe a difference in AR-BSI rates between patients with and without diabetes in univariate or multivariable analysis. Diabetes was found to be a risk factor for bacteraemia in some $[16,18]$ but not all studies [17]. Similarly, gender did not have an effect on infection rates, and this is consistent with multiple prior studies [16-18].

Gram positive organisms were the predominant microbes from positive blood cultures in our cohort, with only a smaller proportion of AR-BSIs attributable to Gram negative (GN) organisms. Whereas studies from the US, Brazil, Greece and Singapore reported GN bacterial growth in 15 to $26 \%$ of positive cultures [17, 18, 24, 27, 33, 34], GN bacteria were identified in less than $5 \%$ of specimens in our study. CoNS were identified in more than $60 \%$ of cases, 
which is a higher rate than compared to published literature. CoNS, despite being common constituents of the normal flora of the skin, can be major nosocomial pathogens and cause significant morbidity in patients with CVCs [35]. Their spread is facilitated by poor hand hygiene and inadequate disinfection or sterilisation of instruments or surfaces [35]. It is difficult however to compare frequencies between different studies because of different study inclusion and exclusion criteria, and different definitions used. Our study also highlights the high burden of these events on both the patient and the health system. Two bacteraemia-related fatalities were identified. The majority of patients with AR-BSIs required hospitalisation, and catheter replacement was required in more than $50 \%$ of patients.

A few limitations are worth mentioning. The study was retrospective in design and thus not all known risk factors were measured at baseline. Patients receiving dialysis by CVC may be inherently different to those with AVF. Our data did not enable us to characterise patients beyond the variables used in our models. We did not capture exit-site and tunnel infections in our study. However, it should be noted that there is subjectivity in the definition of these events and these may or may not be associated with bacteraemia. The study reflects a single centre experience, which may limit generalisability. In addition, we did not differentiate between incident or prevalent HD patients in our study. Therefore, we must acknowledge we were unable to assess whether dialysis/ catheter vintage modifies the relationship with infection risk in those with CVC's. Finally, our unit's policy on management of access-related bacteraemia did not have a standardised protocol to check for clearance of bacteraemia prior to or shortly after discontinuation of the antimicrobial agent. This precluded conducting a reliable comparison of clearance duration. These limitations, however, were counterbalanced by several strengths. First, our study included all chronic HD patients who received dialysis at a large centre, and none of the patients had missing data. Second, all microbiology test results were available from a single central laboratory ensuring consistency and reliability of reporting. This was of concern in data reported from dialysis facilities in the US, as blood cultures were analysed at several different laboratories, particularly for samples obtained after hospitalisation $[24,36]$. We reported on BSI, a measure that is based on an objective test, using standard definitions. Finally, the period of follow-up was long relative to other published studies in the literature.

\section{Conclusions}

AR-BSI remain a significant complication particularly among contemporary cohorts of patients undergoing haemodialysis by tunnelled catheters. The risk is present for all subgroups including the elderly. Access-related bloodstream infections impose a huge burden on patients and on health systems. Active surveillance of BSI linked to quality improvement initiatives should remain an integral part of all dialysis programmes to reduce catheter- associated infections and improve patient outcomes.

\section{Additional file}

Additional file 1: Table S1. Number of patients and type of access in each month in the observation period. Table S2. Number of patient months in total and in 2015, 2016 by access type. Table S3. Baseline characteristics by age group in the whole study population. (DOC 83 kb)

\section{Abbreviations}

AR-BSI: Access-related bloodstream infections; AVF: Arterio-venous fistula; CDC: Centers for Disease Control and Prevention; Cl: Confidence intervals; CoNS: Coagulase-negative Staphylococci; CRBSIs: Catheter-related

Bloodstream Infections; CRE: Carbapenem-resistant Enterobacteriaceae; CVC: Central venous catheter; ESBL: Extended spectrum beta lactamase; ESKD: End-stage kidney disease; GN: Gram-negative; HD: Haemodialysis; IQR: Inter-quartile ranges; KDCPMS: Kidney Disease Clinical Patient Management System; MRSA: Methicillin-resistant Staphylococcus aureus; NHSN: National Healthcare Safety Network; RR: Risk ratio; VRE: Vancomycinresistant Enterococcus species

\section{Acknowledgements}

We would like to thank James Powell, Microbiology Surveillance Scientist at University Hospital Limerick for his help in retrieving blood culture results.

\section{Funding}

A.G.S was supported by grants from the Irish Heart Foundation, Midwest Kidney Disease Research and Education Foundation (MKid), Limerick and the Health Research Institute, University of Limerick. A.G.S and L.D.B were supported by grants from the Health Research Board of Ireland (HRA2013-PHR-685 and HRA-2013-PHR-437). A.G.S. has consulted for Amgen and Vifor Pharma. The other authors have no relevant financial relationships to disclose.

\section{Availability of data and materials}

The data that support the findings of this study are available from authors but restrictions apply to the availability of these data, which were used under license for the current study, and so are not publicly available. Data are however available from the authors upon reasonable request and with permission of University of Limerick and Limerick University Hospital, Ireland.

\section{Authors' contributions}

Research idea and project design: WH, LB, NO, LC, AS. Data collection and dataset setup: WH, HM, AA. Data analysis: WH, LB. Manuscript editing: HM, $W H, L B, N O, L C, A S$. All authors contributed to the development of the manuscript, and approved the final version.

Ethics approval and consent to participate

Ethical approval was not sought this study as the surveillance of infections in dialysis patients is part of regular clinical audit and the hospital's quality improvement programme [26].

Consent for publication Not applicable

\section{Competing interests}

Professor Austin Stack serves on the Editorial Board of BMC Nephrology, but did not participate in the review of this manuscript. Other authors had no disclosures to declare. 


\section{Publisher's Note}

Springer Nature remains neutral with regard to jurisdictional claims in published maps and institutional affiliations.

\section{Author details}

'Division of Nephrology, Department of Medicine, University Hospital Limerick, Limerick, Ireland. ${ }^{2}$ Graduate Entry Medical School, University of Limerick, Limerick, Ireland. ${ }^{3}$ Division of Microbiology, Department of Medicine, University Hospital Limerick, Limerick, Ireland. ${ }^{4}$ Health Research Institute, University of Limerick, Limerick, Ireland.

\section{Received: 10 September 2018 Accepted: 11 February 2019} Published online: 26 February 2019

\section{References}

1. Skov Dalgaard L, Norgaard M, Jespersen B, Jensen-Fangel S, Ostergaard LJ, Schonheyder HC, Sogaard OS. Risk and prognosis of bloodstream infections among patients on chronic hemodialysis: a population-based cohort study. PLoS One. 2015;10(4):e0124547

2. Coresh J, Selvin E, Stevens LA, Manzi J, Kusek JW, Eggers P, Van Lente F, Levey AS. Prevalence of chronic kidney disease in the United States. Jama. 2007:298(17):2038-47.

3. Ravani P, Palmer SC, Oliver MJ, Quinn RR, MacRae JM, Tai DJ, Pannu NI, Thomas C, Hemmelgarn BR, Craig JC, et al. Associations between hemodialysis access type and clinical outcomes: a systematic review. J Am Soc Nephrol. 2013:24(3):465-73.

4. Albuquerque SE, Cavalcante Rde S, Ponce D, Fortaleza CM. Epidemiology of healthcare-associated infections among patients from a hemodialysis unit in southeastern Brazil. Braz J Infect Dis. 2014;18(3):327-30.

5. Methven S, Steenkamp R, Fraser S: UK renal registry 19th annual report: chapter 5 survival and causes of death in UK adult patients on renal replacement therapy in 2015: national and Centre-specific analyses. Nephron 2017, 137 Suppl 1:117-150.

6. Vassalotti JA, Jennings WC, Beathard GA, Neumann M, Caponi S, Fox CH, Spergel LM. Fistula first breakthrough initiative: targeting catheter last in fistula first. Semin Dial. 2012;25(3):303-10.

7. Navuluri R, Regalado S. The KDOQI 2006 vascular access update and fistula first program synopsis. Semin Interv Radiol. 2009;26(2):122-4.

8. Jindal K, Chan CT, Deziel C, Hirsch D, Soroka SD, Tonelli M, Culleton BF. Hemodialysis clinical practice guidelines for the Canadian Society of Nephrology. J Am Soc Nephrol. 2006;17(3 Suppl 1):S1-27.

9. Renal Association Vascular Access for Haemodialysis Clinical Practice Guideline https://renal.org/wp-content/uploads/2017/06/vascular-access.pdf. Accessed 16 Feb 2019.

10. Napalkov P, Felici DM, Chu LK, Jacobs JR, Begelman SM. Incidence of catheter-related complications in patients with central venous or hemodialysis catheters: a health care claims database analysis. BMC Cardiovasc Disord. 2013:13:86

11. Lacson E, Wang W, Lazarus JM, Hakim RM. Change in vascular access and hospitalization risk in long-term hemodialysis patients. Clin J Am Soc Nephrol. 2010;5(11):1996-2003.

12. McCann M, Clarke M, Mellotte G, Plant L, Fitzpatrick F. Vascular access and infection prevention and control: a national survey of routine practices in Irish haemodialysis units. Clin Kidney J. 2013;6(2):176-82.

13. Hussein WF, Mohammed H, Browne L, Plant L, Stack AG. Prevalence and correlates of central venous catheter use among haemodialysis patients in the Irish health system - a national study. BMC Nephrol. 2018;19(1):76.

14. Pisoni RL, Zepel L, Port FK, Robinson BM. Trends in US vascular access use, patient preferences, and related practices: an update from the US DOPPS practice monitor with international comparisons. Am J Kidney Dis. 2015; 65(6):905-15.

15. Miller LM, Clark E, Dipchand C, Hiremath S, Kappel J, Kiaii M, Lok C, Luscombe R, Moist L, Oliver M, et al. Hemodialysis tunneled catheter-related infections. Can. J. Kidney Health Dis. 2016:3:2054358116669129.

16. Wang K, Wang P, Liang X, Lu X, Liu Z. Epidemiology of haemodialysis catheter complications: a survey of 865 dialysis patients from 14 haemodialysis centres in Henan province in China. BMJ Open. 2015; 5(11):e007136.

17. Murea M, James KM, Russell GB, Byrum GV 3rd, Yates JE, Tuttle NS, Bleyer AJ, Burkart JM, Freedman BI. Risk of catheter-related bloodstream infection in elderly patients on hemodialysis. Clin J Am Soc Nephrol. 2014;9(4):764-70.
18. Fysaraki M, Samonis G, Valachis A, Daphnis E, Karageorgopoulos DE, Falagas ME, Stylianou K, Kofteridis DP. Incidence, clinical, microbiological features and outcome of bloodstream infections in patients undergoing hemodialysis. Int J Med Sci. 2013;10(12):1632-8.

19. Richardson Al 2nd, Leake A, Schmieder GC, Biuckians A, Stokes GK, Panneton JM, Glickman MH. Should fistulas really be first in the elderly patient? J. Vasc. Access. 2009;10(3):199-202.

20. Hoen B, Paul-Dauphin A, Hestin D, Kessler M. EPIBACDIAL: a multicenter prospective study of risk factors for bacteremia in chronic hemodialysis patients. J Am Soc Nephrol. 1998;9(5):869-76.

21. Tokars JI, Light P, Anderson J, Miller ER, Parrish J, Armistead N, Jarvis WR, Gehr T. A prospective study of vascular access infections at seven outpatient hemodialysis centers. Am J Kidney Dis. 2001;37(6):1232-40.

22. Taylor G, Gravel D, Johnston L, Embil J, Holton D, Paton S. Incidence of bloodstream infection in multicenter inception cohorts of hemodialysis patients. Am J Infect Control. 2004;32(3):155-60.

23. Quittnat Pelletier F, Joarder M, Poutanen SM, Lok CE. Evaluating approaches for the diagnosis of hemodialysis catheter-related bloodstream infections. Clin J Am Soc Nephrol. 2016;11(5):847-54.

24. Nguyen DB, Shugart A, Lines C, Shah AB, Edwards J, Pollock D, Sievert D, Patel PR. National Healthcare Safety Network (NHSN) dialysis event surveillance report for 2014. Clin J Am Soc Nephrol. 2017;12(7):1139-46.

25. Centers for Disease Control and Prevention: Dialysis Event Protocol http:// www.cdc.gov/nhsn/PDFs/pscManual/8pscDialysisEventcurrent.pdf. Accessed 16 Feb 2019.

26. Research Ethics Committees https://www.hse.ie/eng/services/list/5/ publichealth/publichealthdepts/research/rec.html. Accessed 16 Feb 2019.

27. Patel PR, Shugart A, Mbaeyi C, Goding Sauer A, Melville A, Nguyen DB, Kallen AJ. Dialysis event surveillance report: National Healthcare Safety Network data summary, January 2007 through April 2011. Am J Infect Control. 2016:44(8):944-7.

28. Hymes JL, Mooney A, Van Zandt C, Lynch L, Ziebol R, Killion D. Dialysis catheter-related bloodstream infections: a cluster-randomized trial of the ClearGuard HD antimicrobial barrier cap. Am J Kidney Dis. 2017;69(2):220-7.

29. Yi SH, Kallen AJ, Hess S, Bren VR, Lincoln ME, Downham G, Kelley K, Booth $S L$, Weirich $H$, Shugart A, et al. Sustained infection reduction in outpatient hemodialysis centers participating in a collaborative bloodstream infection prevention effort. Infect Control Hosp Epidemiol. 2016;37(7):863-6.

30. Lee T. Fistula first initiative: historical impact on vascular access practice patterns and influence on future vascular access care. Cardiovasc Eng Technol. 2017:8(3):244-254

31. Saleh T, Sumida K, Molnar MZ, Potukuchi PK, Thomas F, Lu JL, Gyamlani GG, Streja E, Kalantar-Zadeh K, Kovesdy CP. Effect of age on the Association of Vascular Access Type with mortality in a cohort of incident end-stage renal disease patients. Nephron. 2017;137(1):57-63.

32. Lee $T$, Thamer $M$, Zhang $Q$, Zhang $Y$, Allon M. Vascular access type and clinical outcomes among elderly patients on hemodialysis. Clin J Am Soc Nephrol. 2017:12(11):1823-30.

33. Fram D, Okuno MF, Taminato M, Ponzio V, Manfredi SR, Grothe C, Belasco A Sesso R, Barbosa D. Risk factors for bloodstream infection in patients at a Brazilian hemodialysis center: a case-control study. BMC Infect Dis. 2015;15:158.

34. Loo LW, Liew YX, Choong HL, Tan AL, Chlebicki P. Microbiology and audit of vascular access-associated bloodstream infections in multi-ethnic Asian hemodialysis patients in a tertiary hospital. Infectious diseases (London, England). 2015;47(4):225-30

35. Becker K, Heilmann C, Peters G. Coagulase-negative staphylococci. Clin Microbiol Rev. 2014;27(4):870-926.

36. Nguyen DB, See I, Gualandi N, Shugart A, Lines C, Bamberg W, Dumyati G, Harrison LH, Lesher L, Nadle J, et al. Completeness of methicillin-resistant Staphylococcus aureus bloodstream infection reporting from outpatient hemodialysis facilities to the National Healthcare Safety Network, 2013. Infect Control Hosp Epidemiol. 2016;37(2):205-7. 